\title{
Partially incoherent optical vortices in self-focusing nonlinear media
}

\author{
Chien-Chung Jeng ${ }^{1}$, Ming-Feng Shih ${ }^{1}$, Kristian Motzek $^{2,3}$, and Yuri Kivshar ${ }^{3}$ \\ ${ }^{1}$ Physics Department, National Taiwan University, Taipei, 106, Taiwan \\ 2 Institute of Applied Physics, Darmstadt University of Technology, D-64289 Darmstadt, Germany \\ 3 Nonlinear Physics Group, Research School of Physical Sciences and Engineering, \\ Australian National University, Canberra ACT 0200, Australia
}

\begin{abstract}
We observe stable propagation of spatially localized single- and double-charge optical vortices in a self-focusing nonlinear medium. The vortices are created by self-trapping of partially incoherent light carrying a phase dislocation, and they are stabilized when the spatial incoherence of light exceeds a certain threshold. We confirm the vortex stabilization effect by numerical simulations and also show that the similar mechanism of stabilization applies to higher-order vortices.
\end{abstract}

PACS numbers:

Vortices are fundamental localized objects which appear in many branches of physics, ranging from liquid crystals to superfluids and Bose-Einstein condensates [1]. In optics, vortices are associated with phase dislocations (or phase singularities) carried by diffracting optical beams [2]. When such vortices propagate in selfdefocusing nonlinear media, the vortex core with a phase dislocation becomes self-trapped, and the resulting stationary singular beam is known as an optical vortex soliton [3]. Such vortex solitons have been generated experimentally (e.g. by using a phase mask) within broad diffracting beams in different types of defocusing nonlinear media [4, 5, 6, 7, 8]. Optical vortex solitons demonstrate many common properties with the vortices observed in superfluids and, more recently, in Bose-Einstein condensates [9].

In contrast, optical vortices become highly unstable in self-focusing nonlinear media. Indeed, when a ringlike optical beam with zero intensity at the center and a phase singularity [10] propagates in a self-focusing nonlinear medium, it always decays into several fundamental solitons flying off the main ring [11]. This effect has been observed experimentally in different nonlinear systems, including the saturable Kerr-like nonlinear media 12], biased photorefractive crystals [7], and quadratic nonlinear media [13] in the self-focusing regime. This effect has also been predicted to occur in many other physical systems including attractive Bose-Einstein condensates [14].

A number of recent theoretical papers 15], including the rigorous studies of the vortex stability [16], suggest that the stable propagation of spatial and spatiotemporal vortex-like stationary structures may become possible in the models with competing nonlinearities in the presence of a large higher-order defocusing nonlinearity, but no realistic physical systems to support these theoretical findings have been found so far. Thus, the main question remains open: Can stable optical vortices readily be observed in self-focusing nonlinear media?

The main purpose of this Letter is to demonstrate, for the first time to our knowledge, that stable propagation of spatially localized optical vortices in a self-focusing photorefractive crystal can indeed be observed provided such vortices are created by partially incoherent light car- rying a phase dislocation. In particular, we show, both experimentally and theoretically, that single- and doublecharge optical vortices can be stabilized in self-focusing nonlinear media when the value of the spatial incoherence of light exceeds a certain threshold, and these vortices are readily observed in experiment as stationary self-trapped structures propagating for many diffraction lengths.

We should mention that the generation and properties of singular optical beams created by partially incoherent light is an important issue which is a subject of a current active research even in linear optics (see, e.g, Ref. 17 and references therein). Our results demonstrate that partially incoherent singular beams can exist in self-focusing nonlinear media being stabilized by the light incoherence effect.

First, we present our experimental results. The main purpose of our experiments conducted for a biased photorefractive medium is to generate partially incoherent vortices and vortex solitons, and then inspect their stability in such a self-focusing nonlinear medium. Being driven by the earlier results of Anastassiou et al. 18] who demonstrated the suppression of modulational instability for the stripe spatial solitons created by partially incoherent light, we try to understand whether the other type of the nonlinearity-driven instability, the so-called azimuthal instability, can be suppressed by reducing the coherent properties of optical vortex beams.

The experiment (the setup is shown in Fig. (1) is conducted with a biased photorefractive SBN crystal ( $\mathrm{a} \times \mathrm{b}$ $\mathrm{x} \mathrm{c}=5 \mathrm{~mm} \times 10 \mathrm{~mm} \times 5 \mathrm{~mm})$. First, a cw laser light beam (at $488 \mathrm{~nm}$ ) of the extraordinary polarization is made partially incoherent by passing it through a lens and then through a rotating diffuser. The rotating diffuser introduces random-varying phase and amplitude on the light beam every $1 \mu \mathrm{s}$, which is much shorter than the response time (about $1 \mathrm{~s}$ ) of the crystal under our experimental light illumination. By adjusting the position of the diffuser to near (away from) the focal point of the lens in front the diffuser, we can increase (decrease) the degree of the light coherence. We collect the light after the rotating diffuser by a second lens and then pass is through a computer-generated hologram to imprint a vortex phase (with a single-, for $m=1$, or double-, for 


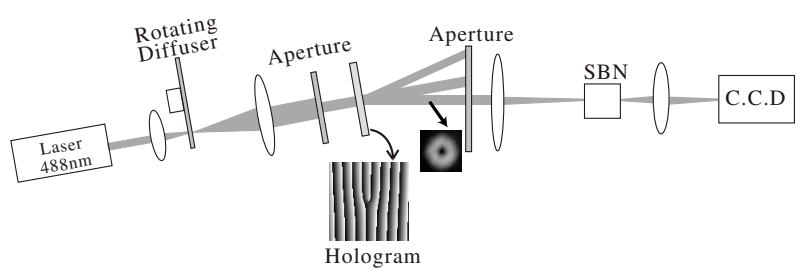

FIG. 1: Schematic of the experimental setup for the observation of the partially incoherent optical vortices; SBN: Strontium Barium Niobate crystal.

$m=2$, charge) on the light beam. Since the partially incoherent light beam can be considered as a superposition of many mutually-incoherent light beams, the first-order diffracted light beam after the hologram becomes a superposition of many mutually-incoherent vortex beams. We then focus and launch the partially coherent vortex beam into the SBN crystal along its a-axis. The crystal is illuminated by a halogen lamp from its top side as necessary for photorefractive screening solitons. The total power of the vortex beam is of $0.17 \mu \mathrm{W}$, which results in the nonlinearity of the photorefractive crystal falling into the Kerr region for the peak intensity of the vortex beam to the background intensity is much less than unity. Then, a lens is used to project the images at the input and output faces onto a CCD camera.

Before showing that reducing the degree of coherence can stabilize the vortex beam propagating in a selffocusing medium, we reproduce the experiment that the coherent single-charge $(m=1)$ vortex light beam cannot stably propagate in a self-focusing medium [7, 12]. We first remove the diffuser from the experimental setup. The vortex beam at the input face of the crystal is shown as Fig. 2(a). With zero biasing voltage, Fig. 2(b) shows the natural diffraction of the vortex light beam. While a $2.5 \mathrm{kV}$ biasing voltage is applied on the photorefractive crystal creating a Kerr-type self-focusing nonlinear medium, the vortex beam breaks up into two pieces [Fig. 2(c)]. This vortex break-up observed in a self-focusing medium is due to the azimuthal instability, and it has been theoretically and experimentally demonstrated previously [11, 12]. We then put back the rotating diffuser and adjust it to a suitable position. The degree of coherence of the vortex light beam can be estimated by the speckle size at the input face of the crystal [Fig. 22 (d)] when we stop the diffuser from rotating. As a voltage of $2.5 \mathrm{kV}$ is applied on the crystal, Fig. 2( g) clearly shows that the vortex light beam is stabilized by the reduction of the degree of coherence though two very unclear bright spots still can be seen on the opposite sides (top and bottom) of the ring-like intensity distribution. As we move the rotating diffuser further away from the focal point of the lens to make the light more incoherent [indicated by Fig. 2(h)] and apply a voltage of $2.5 \mathrm{kV}$ on the crystal, Fig. 2 (k) shows the generated stable partially incoherent

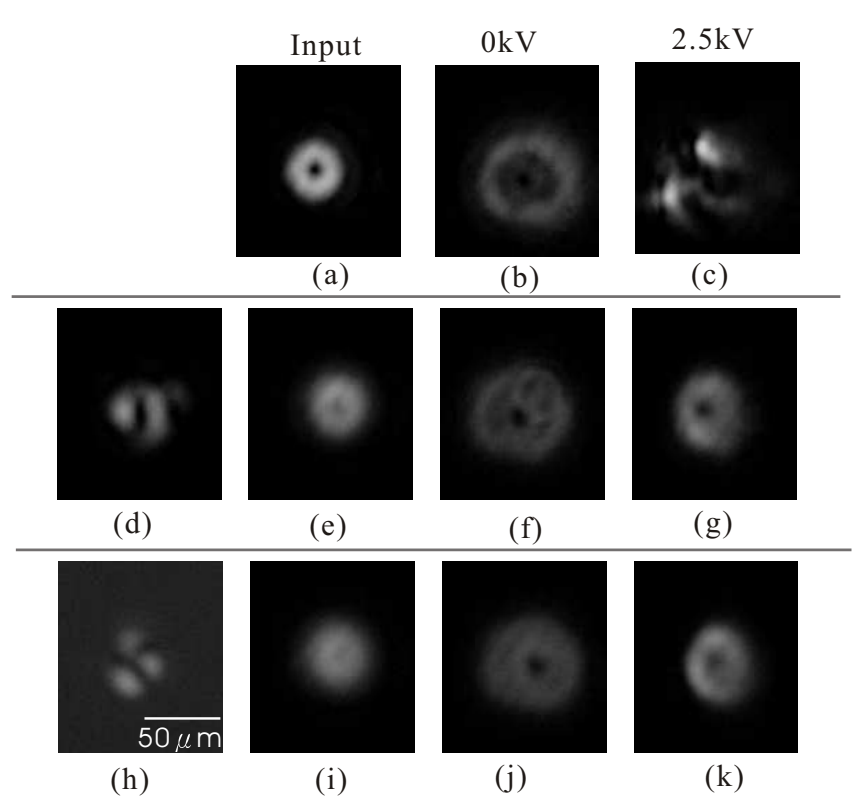

FIG. 2: Experimental results for the intensity distribution of a single-charge vortex beam $(m=1)$ for different degrees of coherence (top: coherent, middle: less coherent, bottom: least coherent). At input face, (a) is coherent light, (e) and (i) are partially incoherent, but (i) is more incoherent than (e) by comparing the speckle pattern shown in (d) and (h). At output face, (b) (f) and (j) show the diffraction when the nonlinearity is off, and (c), (g) and (k) shows the incoherence stabilize the vortex soliton when voltage of $2.5 \mathrm{kV}$ is applied.

vortex soliton at the output face of the crystal.

We notice that the vortex stabilization by the light incoherence can possibly be explained by employing simple physics. Indeed, after some propagation of the partially incoherent beam with an imprinted phase, we observe that the intensity at the center of the beam does not vanish. This means that the incoherent vortex can be decomposed into many mutually incoherent vortex beams with not only their phases randomly distributed but also their central positions offset from each others. In this way, the core of a composite vortex beam will be filled by some light, and the index change at the beam center will become nonzero. We believe that this effect contributes strongly to the vortex stabilization.

We continue with the experiments for the doublecharge vortex beams generated by a phase mask with the $m=2$ dislocation. Figures 3(a-c) show that a coherent double-charge vortex light beam cannot stably propagate in a self-focusing medium (we applied $3 \mathrm{kV}$ here) as well, and it breaks up into pieces as been observed in a self-focusing atomic vapor 12]. When we make the light more incoherent, the double-charge vortex becomes more stable, as shown in Figs. $3(\mathrm{~g})$ and (k). However, we could not make a double-charge vortex soliton here. The double-charge vortex beam diffracts more than a singlecharge vortex beam and, therefore, it requires higher non- 


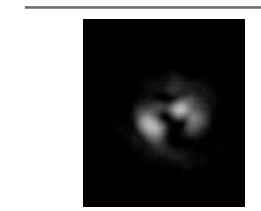

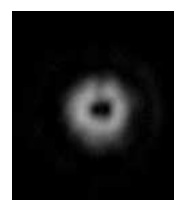

(a)

(d)

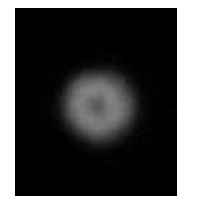

(e)

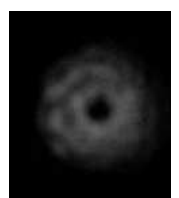

(b)

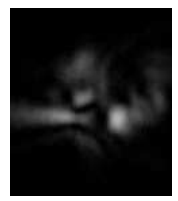

(c)

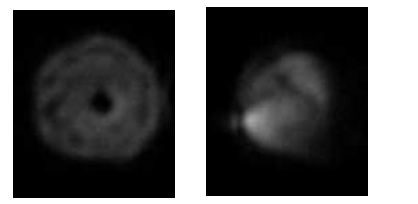

(f)

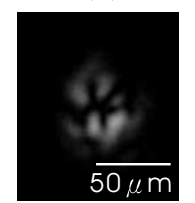

(h)

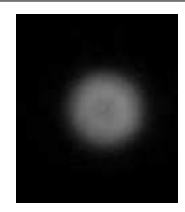

(i) $(\mathrm{g})$

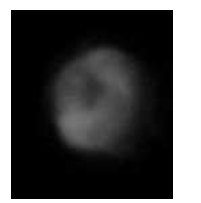

(k)

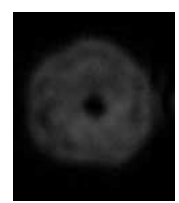

(j)
FIG. 3: Experimental results for a double-charge vortex beam $(m=2)$ for different degrees of coherence (top: coherent, middle: less coherent, bottom: least coherent). At input face, (a) is coherent light, (e) and (i) are partially incoherent, but (i) is more incoherent than (e) by comparing the speckle pattern shown in (d) and (h). At output face, (b) (f) and (j) show the diffraction when the nonlinearity is off, and (c), (g) and (k) shows the incoherence stabilize the vortex soliton when voltage of $3 \mathrm{kV}$ is applied.

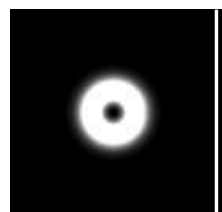

Omm

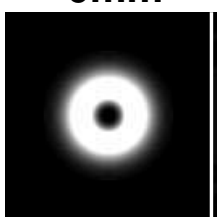

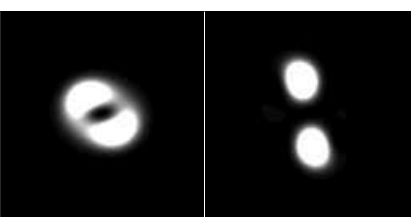

$8 \mathrm{~mm}$

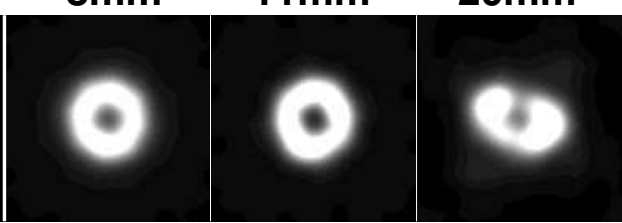

FIG. 4: Numerical results showing the comparison between the evolution of the vortex created by a coherent (upper row) and partially incoherent (lower row) light $\left(\theta_{0}=0.38\right)$. The vortex stabilization by partial incoherence is clearly seen.

linearity (or higher voltage) to form the vortex soliton, but our crystal begins to arc at $3.5 \mathrm{kV}$. Nevertheless, these two experiments show that the reduction of the degree of coherence of the light beam can indeed stabilize the single- or double-charge (or even higher-charge) vortex solitons propagating in a self-focusing medium.

We have also studied numerically the propagation of partially incoherent optical vortices in a photorefractive medium, employing the coherent density ap-
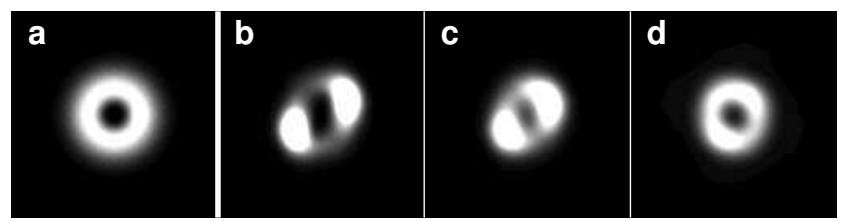

FIG. 5: Numerical results showing the stabilization of the vortex with growing incoherence: (a) input intensity, (b) vortex after $9 \mathrm{~mm}$ of propagation for the coherent case, (c) vortex after $9 \mathrm{~mm}$ for the partially incoherent case, $\theta_{0}=0.14$, and $(\mathrm{d})$ vortex after $9 \mathrm{~mm}$ for the partially incoherent case, $\theta_{0}=0.29$.

proach [18, 19]. The coherent density approach is based on the fact that partially incoherent light can be described by a superposition of mutually incoherent light beams that are tilted with respect to the $z$-axis at different angles. One thus makes the ansatz that the partially incoherent light consists of many coherent, but mutually incoherent light beams $E_{j}: I=\sum_{j}\left|E_{j}\right|^{2}$. By setting $\left|E_{j}\right|^{2}=G(j \vartheta) I$, where

$$
G(\theta)=\frac{1}{\sqrt{\pi} \theta_{0}} e^{-\theta^{2} / \theta_{0}^{2}}
$$

is the angular power spectrum, one obtains a partially incoherent light beam whose coherence is determined by the parameter $\theta_{0}$, i.e. less coherence means bigger $\theta_{0}$. Here, $j \vartheta$ is the angle at which the $j$-th beam is tilted with respect to the $z$-axis. For our simulations we used a set of 1681 mutually incoherent vortices, all initially tilted at different angles. To simulate the photorefractive nonlinearity we use a simple model which predicts that the refractive index change is approximately given by $I /(1+I)$.

Figure 4 shows a comparison between the propagation of the vortices generated by coherent and partially incoherent light (for $\theta_{0}=0.38$ ). Increasing the incoherence further leads to the case where the vortex beam radiates off a lot of its intensity before the azimuthal instability can set in. Larger values of incoherence (i.e. larger values of $\theta_{0}$ ) correspond to a complete suppression of the vortex instability, and this confirms the experimental results presented above.

Figures 5 (a-d) show our numerical results for the propagation of an input Gaussian beam carrying a phase dislocation [(a)] after the total propagation $(9 \mathrm{~mm})$ in a nonlinear medium for the coherent light $[(\mathrm{b})]$ and two different partially incoherent beams $[(\mathrm{c}, \mathrm{d})]$, corresponding to the values $\theta_{0}=0.14$ and $\theta_{0}=0.29$, respectively. The most obvious difference to the scenario of the propagation the a coherent vortex is that the vortex decay undergoes a visible delay when the degree of incoherence grows. Furthermore, in the incoherent case the vortex changes its profile only very slowly as it propagates and thus can be considered as being in a transition stage between the decay and stabilization.

Finally, we have studied numerically the propagation of a double-charge vortex beam in both coherent and 

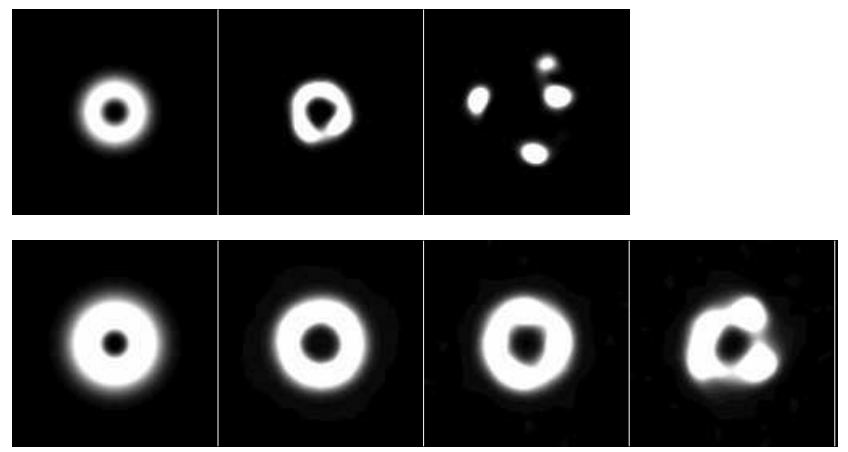

FIG. 6: Propagation of a double-charge vortex in (top row) the coherent case at $z=0,8.6$, and $14.5 \mathrm{~mm}$, and (bottom row) in the partially incoherent case with $\theta_{0}=0.35$ at $z=0$, 8.6, 14.7, and $18.8 \mathrm{~mm}$.

partially incoherent cases, as shown in Fig. 6] The initial perturbation in both cases have been chosen to be very small in order to obtain a clearer picture of the instability, therefore the propagation distances are quite long. In numerics, since we have a possibility to observe long propagation distances, we did not find that the vortices can be completely stabilized by increasing incoherence.
When using the values of $\theta_{0}$ far above the value $\theta_{0}=0.35$ used in Fig. [6] we observe that the vortex just radiates off a lot of its intensity and then decays. The problem is obviously that the vortices are not only incoherent along the angular, but also the radial direction. We believe that the experiment would confirm this observation if a longer crystal was available.

In conclusion, for the first time to our knowledge, we have observed the stable propagation of single- and double-charge optical vortices in a self-focusing nonlinear medium. The vortices have been created by partially incoherent light beams carrying a phase dislocation and propagating in a photorefractive nonlinear medium. The vortex azimuthal instability in a self-focusing nonlinear medium was suppressed for the light incoherence above a critical value. The experimental results have been confirmed by numerical simulations which also provide an insight into the physical mechanisms of the vortex stabilization.

Yuri Kivshar thanks the Physics Department of the Taiwan University for hospitality. This work was supported by a collaboration program between the Australian Academy of Science and the National Science Council of Taiwan, and the Australian Research Council.
[1] L.M. Pismen, Vortices in Nonlinear Fields (Clarendon Press, Oxford, 1999), 290 pp.

[2] See, e.g., a comprehensive review paper, M.S. Soskin and M.V. Vasnetsov, in Progress in Optics, Vol. 42, Ed. E. Wolf (Elsevier, Amstredam, 2001).

[3] See, e.g., Yu.S. Kivshar and G.P. Agrawal, Optical Solitons: From Fibers to Photonic Crystals (Academic, San Diego, 2003), Chap. 8.

[4] G.A. Swartzlander and C. Law, Phys. Rev. Lett. 69, 2503 (1992).

[5] G. Duree, M. Morin, G. Salamo, M. Segev, B. Crosignani, P. Di Porto, E. Sharp, and A. Yariv, Phys. Rev. Lett. 74, 1978 (1995).

[6] J. Christou, V.Tikhonenko, Yu.S. Kivshar, and B. Luther-Davies, Opt. Lett. 21, 1649 (1996).

[7] Z. Chen, M. Shih, M. Segev, D.W. Wilson, R.E. Muller, and P.D. Maker, Opt. Lett. 22, 1751 (1997).

[8] Yu.S. Kivshar and B. Luther-Davies, Phys. Rep. 298, 81 (1998).

[9] See, e.g., K.W. Madison, F. Chevy, W. Wohlleben, and J. Dalibard, Phys. Rev. Lett. 84, 806 (2000); C. Raman, J.R. Abo-Shaeer, J.M. Vohels, and W. Ketterle, Phys. Rev. Lett. 87, 210402 (2001).

[10] Such beams were first suggested in V.I. Kruglov and R.A. Vlasov, Phys. Lett. A 111, 401 (1985).

[11] W.J. Firth and D.V. Skryabin, Phys. Rev. Lett. 79, 2450 (1997); D.V. Skryabin and W. Firth, Phys. Rev. E 58, 3916 (1998).

[12] V. Tikhonenko, J. Christou, and B. Luther-Davies, J. Opt. Soc. Am. B 12, 2046 (1995); Phys. Rev. Lett. 76,
2698 (1996).

[13] D.V. Petrov, L. Torner, J. Nartorell, R. Vilaseca, J.P. Torres, and C. Cojocaru, Opt. Lett. 23, 1444 (1998).

[14] H. Saito and M. Ueda, Phys. Rev. Lett. 89, 190402 (2002).

[15] M. Quiroga-Teixeiro and H. Michinel, J. Opt. Soc. Am. B 14, 2004 (1997); A. Desyatnikov, A. Maimistov, and B.A. Malomed, Phys. Rev. E 61, 3107 (2000); H. Michinel, J. Campo-Táboas, M.L. Quiroga-Teixeiro, J.R. Salqueiro, and R. Gracía-Fernández, J. Opt. B: Quantum Semiclass. 3, 314 (2001); V. Skarka, N.B. Aleksić, V.I. Berezhiani, Phys. Lett. A 291, 124 (2001); B.A. Malomed, L.-C. Crasovan, and D. Mihalache, Physica D 161, 187 (2002); T.A. Davydova, A.I. Yakimenko, and Yu.A. Zaliznyak, Phys. Rev. E 67, 026402 (2003).

[16] I. Towers, A.V. Buryak, R.A. Sammut, B.A. Malomed, L.-C. Crasovan, and D. Mihalache, Phys. Lett. A 288, 292 (2001); Phys. Rev. A 63, 055601 (2001); D. Mihalache, D. Mazilu, L.-C. Crasovan, I. Towers, A.V. Buryak, B.A. Malomed, L. Torner, J.P. Torres, and F. Lederer, Phys. Rev. Lett. 88, 073902 (2002).

[17] G. Gbur and T.D. Visser, Opt. Commun. 222, 117 (2003).

[18] C. Anastassiou, M. Soljacić, M. Segev, E.D. Eugenieva, D.N. Christodoulides, D. Kip, Z.H. Musslimani, and J.P. Torres, Phys. Rev. Lett. 85, 4888 (2000).

[19] D.N. Christodoulides, T.H. Coskun, M. Mitchell, and M. Segev, Phys. Rev. Lett 78, 646 (1997). 